Service social

\title{
La solidarité horizontale chez les personnes âgées. Notes de lecture et de recherche
}

\section{Marc-André Delisle}

Volume 46, numéro 1, 1997

Politique et intervention

URI : https://id.erudit.org/iderudit/706753ar

DOI : https://doi.org/10.7202/706753ar

Aller au sommaire du numéro

\section{Éditeur(s)}

École de service social de l'Université Laval

ISSN

1708-1734 (numérique)

Découvrir la revue

Citer cette note

Delisle, M.-A. (1997). La solidarité horizontale chez les personnes âgées. Notes de lecture et de recherche. Service social, 46(1), 147-173.

https://doi.org/10.7202/706753ar
Résumé de l'article

L'objectif de cet article est d'étayer les assises théoriques et empiriques sous-jacentes au phénomène de la solidarité horizontale chez les aînés. Après avoir forgé le concept de solidarité horizontale (ou intragénérationnelle), l'auteur a résumé quelques théories pouvant expliquer cette réalité. Une synthèse d'études empiriques effectuées sur certains indicateurs montre que la conscience générationnelle existe bien chez les aînés, que la plupart d'entre eux s'identifient à leurs pairs et qu'il y a beaucoup d'entraide parmi eux. Ces phénomènes sont encore plus prégnants quand les personnes âgées vivent dans le même environnement. Toutefois, les échanges se font la plupart du temps de manière informelle ou à l'initiative de certains leaders. Il y a donc des contextes et des sphères dans lesquels la solidarité horizontale se manifeste, mais cette solidarité n'apparaît pas partout. 


\section{La solidarité horizontale chez les personnes âgées Notes de lecture et de recherche}

Marc-André DELISLE

L'objectif de cet article est d'étayer les assises théoriques et empiriques sous-jacentes au phénomène de la solidarité horizontale chez les aînés. Après avoir forgé le concept de solidarité horizontale (ou intragénérationnelle), l'auteur a résumé quelques théories pouvant expliquer cette réalité. Une synthèse d'études empiriques effectuées sur certains indicateurs montre que la conscience générationnelle existe bien chez les aînés, que la plupart d'entre eux s'identifient à leurs pairs et qu'il y a beaucoup d'entraide parmi eux. Ces phénomènes sont encore plus prégnants quand les personnes âgées vivent dans le même environnement. Toutefois, les échanges se font la plupart du temps de manière informelle ou à l'initiative de certains leaders. II y a donc des contextes et des sphères dans lesquels la solidarité horizontale se manifeste, mais cette solidarité n'apparaît pas partout.

This article presents the theoretical and empirical foundations of intra-generational solidarity among elderly people. The paper first deals with the concept of intra-generational or (horizontal) solidarity and then summarizes several theories and empirical studies which may explain and contribute to our understanding of this behavioral pattern. For instance, identification among peers and mutual aid between elderly people occur frequently, in particular if they share the same environment. However, these social interactions are, in general, informal or are initiated by a few leaders. These interactions do not occur universally : intra-generational solidarity is observed more frequently in certain situations and social contexts. 


\section{INTRODUCTION}

Cet article porte sur la solidarité horizontale chez les aînés. II a été rédigé dans le but d'approfondir l'étude du concept de solidarité horizontale et de voir si ce concept peut contribuer à une meilleure compréhension des rapports d'entraide au sein de cette population. La problématique de la solidarité horizontale est cruciale dans le contexte actuel, marqué par le désengagement de l'État ainsi que par le transfert de la responsabilité du maintien à domicile des personnes âgées vers les aidants naturels et les groupes communautaires. L'idée sous-jacente à cette problématique est que, si les aînés s'entraident davantage, ils auront moins besoin des adultes plus jeunes et de l'État. Dès lors, les gens d'âge actif seront soulagés de l'obligation de soutenir la génération qui les précède et l'Etat pourra faire des économies substantielles. Cette idée est à l'origine de divers projets favorisant le soutien mutuel entre aînés (France et McDowell, 1982; Riessman, 1982; Scharlach, 1988). Quelques-uns de ces projets ont connu un succès indéniable, mais peu d'entre eux ont été évalués scientifiquement. De plus, on n'a guère étudié la logique de la solidarité horizontale. Tel est l'objet de cet article. Nous tenterons d'apporter des éléments de réponse aux interrogations suivantes :

A) Quelles sont les assises théoriques du concept de solidarité horizontale?

B) Que révèlent les recherches empiriques effectuées pour mesurer l'importance de la solidarité horizontale chez les aînés?

Après avoir défini dans la première section les concepts de solidarité, de génération, d'horizontalité et de verticalité, pour en arriver à forger celui de solidarité horizontale (ou intragénérationnelle), nous ferons un survol de quelques théories pouvant expliquer ce phénomène. Ensuite (troisième section), nous effectuerons une synthèse d'études empiriques effectuées sur certains indicateurs de la solidarité horizontale chez les gens âgés. En terminant, nous résumerons notre propos et nous proposerons des pistes de recherche. 


\section{LES CONCEPTS FONDAMENTAUX}

Dans cette section, nous examinerons successivement les notions de solidarité et de cohésion sociale, de génération, puis d'horizontalité et de verticalité.

\section{Solidarité et cohésion sociale}

Selon Le Robert (1990, p. 1829), le mot « solidaire » vient du latin juridique in solidum qui veut dire "pour le tout». La solidarité serait alors une "relation entre personnes ayant conscience d'une communauté d'intérêts, qui entraîne, pour les unes, l'obligation morale de ne pas desservir les autres et de leur porter assistance » (ibid.). "Cette notion se fonde donc sur le constat d'une interdépendance entre les individus et sur les obligations qui en découlent » (Guillemard, 1989, p. 186). Pour Willems et Cuvillier (1970, p. 268-269), la solidarité sociale est synonyme de cohésion sociale. La solidarité sociale est alors tributaire du "degré d'intégration de l'agrégat » (ibid.). D'après ces auteurs, l'intégration sociale est un « Ajustement réciproque de différents groupes [et individus] leur permettant de former une société organisée ». Cet ajustement est l'aboutissement d'un « processus d'intériorisation qui permet à un individu de réagir conformément aux normes, valeurs qui régissent le groupe » (Brémond et Geledan, 1981, p. 217). Pour qu'il y ait intégration, cohésion et solidarité sociales, il faut un certain consensus dans le groupe, ce concept étant défini par Willems et Cuvillier (ibid.). comme étant la "Conformité de pensées, de sentiments et d'actes qui caractérise les composants d'un groupe ou d'une société déterminés ».

Selon Rocher (1969, tome 1, p. 68), le partage des valeurs est une condition sine qua non pour que se réalise tout consensus et pour qu'il y ait intégration, cohésion et solidarité sociales, car les individus s'approprient les valeurs de leur groupe, qui deviennent alors une composante fondamentale de leur propre identité sociale. Les valeurs sont donc source de solidarité, mais en même temps elles peuvent entraîner le rejet ou l'exclusion de ceux qui n'y souscrivent pas.

Le lien entre solidarité et cohésion a également été souligné par les psychologues sociaux. Ainsi, pour Mudrack (1989, p. 39), " un groupe cohésif est un groupe qui se tient solidement - celui dont les membres sont liés les uns aux autres et à 
l'ensemble du groupe » (traduction libre $\left.{ }^{1}\right)$. L'auteur affirme également que la cohésion se traduit par des sentiments de solidarité, par l'harmonie entre les membres du groupe et par l'engagement envers le groupe. Les relations entre les membres d'un groupe cohésif sont fortes, de sorte qu'il exerce un attrait sur ses membres et peut même attirer ceux qui n'en font pas partie (voir aussi Guimond, 1994). Cette perspective rejoint celle de Bengtson et Schrader (1982, p. 118 et s.). Pour eux, la solidarité est la résultante de phénomènes psychologiques et sociaux qui retiennent les individus dans le groupe et qui les amènent à interagir. Certaines de ces forces sont d'origine macrosociale (les ressemblances, la division du travail), comme l'a montré Durkheim (1978); d'autres ont trait à la similitude des besoins, à la convergence des buts poursuivis ou, encore, à l'attirance exercée par les membres du groupe les uns sur les autres, de même qu'à l'attrait du groupe sur ses membres.

Bref, la solidarité est le produit de facteurs sociaux et psychosociaux. Les facteurs sociaux favorisent l'émergence de communautés de pensée et d'intérêt entre les membres du groupe, alors que les facteurs psychosociaux sont à l'origine de sentiments positifs entre les individus qui le composent. De plus, le rapport de solidarité est, en principe, exclusif ou privilégié. II peut quand même y avoir des échanges avec des tiers, mais ces échanges ne doivent pas avoir préséance sur les relations qu'entretiennent les membres d'un groupe se voulant solidaire.

\section{Le concept de génération}

Autre concept fondamental, celui de génération, souvent employé comme synonyme de cohorte. En fait, ces deux notions sont parentes, mais leur signification sociologique est distincte. Elles désignent des individus nés à la même époque, donc ayant sensiblement le même âge. Cependant, la cohorte est essentiellement une catégorie sociale, c'est-à-dire un ensemble d'individus sans liens entre eux. Elle est formée de gens nés pendant une même période ou qui ont commencé une activité en même temps (ex. : cohorte d'étudiants).

1. "A cohesive group is one that "sticks together" - one whose members are "bonded" to one another and to the group as a whole. » 
Le concept de génération a d'autres significations. En plus d'être synonyme de cohorte, il peut désigner:

a) un rapport de filiation. Par exemple: la génération des parents et celle des enfants. Dans ce cas, les individus n'ont pas exactement le même âge;

b) une période historique de 20 ou 30 ans qui correspond au temps nécessaire pour renouveler la direction d'une société;

c) des gens sensiblement du même âge qui ont partagé des expériences ayant façonné leur mode de vie et leur manière de penser. Par exemple : la génération de la Crise des années trente; celle de la récession des années quatre-vingt, etc. (Attias-Donfut, 1991, p. 58-59).

Dans ce dernier cas, les individus sont censés être conscients de ce qui les distingue de leurs prédécesseurs et de leurs successeurs (Attias-Donfut, 1988; Girardet, 1983; Mannheim, 1952). Cela les prédispose à concevoir des formes de solidarité, tout comme ceux qui appartiennent à une même classe sociale. Or, l'idée de la solidarité horizontale repose justement sur le principe voulant que les membres d'une génération soient portés à s'identifier les uns aux autres et à entretenir des liens privilégiés parce qu'ils font partie d'un même univers socioculturel. $\mathrm{Ce}$ principe est issu de la pensée de K. Mannheim, sociologue allemand (1893-1947) qui a écrit un texte fondamental pour la sociologie des générations (Mannheim, 1952; voir aussi AttiasDonfut, 1988, 1991 et 1993 ainsi que Scott et Grasmick, 1979). Dans ce texte, Mannheim constate tout d'abord que la génération n'est pas un groupe concret au même titre qu'une association, une famille ou une tribu. Cependant, la génération est constituée d'individus qui sont susceptibles de développer des affinités et d'adhérer à des valeurs communes du fait qu'ils ont été influencés au même âge par les courants culturels de leur époque. Cela favorise l'émergence d'une conscience générationnelle permettant la formation "d'unités de génération » ayant tous les attributs et les caractéristiques des groupes concrets (AttiasDonfut, 1991, p. 26). Ce principe, pour logique qu'il soit, est critiquable à maints égards, comme le souligne Girardet (1983), et son bien-fondé a été testé empiriquement. Avant d'évoquer les recherches qui ont permis de vérifier cette proposition, il importe de préciser les notions d'horizontalité et de verticalité, puis de discuter de la manière dont elles sont employées en sciences sociales. 


\section{Horizontalité et verticalité}

Les concepts de solidarité horizontale et verticale sont définis de façon géographique, sociale et générationnelle dans les études qui ont marqué le développement des sciences sociales auX $X I X^{\mathrm{e}}$ et $X X^{\mathrm{e}}$ siècles (Boudon [dir.], 1989; Rocher, 1969). Ces concepts sont tout d'abord apparus dans les études sur les communautés. Pour Warren (1963, p. 237), une communauté est un système social local (localized social system) constitué d'unités reliées entre elles par des schèmes (pattern) d'interaction. La communauté est donc un microcosme (ou une microsociété) formé de groupes et d'individus qui entretiennent deux types de relations : des interrelations (entre eux) et des rapports avec le reste de la société. Dans les études de communautés, le critère de différenciation entre les rapports horizontaux et verticaux est géographique. Ainsi, les interrelations s'actualisent au niveau local, c'est-à-dire à l'intérieur même de la communauté; c'est pourquoi les auteurs consultés les qualifient d'«horizontales ». Williams (1983) inclut dans les interrelations horizontales tous les schèmes d'interaction que développent les organismes et institutions du milieu ainsi que les résidants d'une communauté. Cela englobe les rapports formels entre les groupes qui agissent dans la localité et les relations informelles entre les individus. Dès lors, sont définis comme interactions horizontales tout autant l'entraide entre les membres d'une famille dans un milieu donné que le soutien apporté par les organismes communautaires aux résidants d'une localité.

Warren et Williams opposent ce type de rapports à ceux qu'ont les groupes et individus avec leurs homologues demeurant en dehors de la communauté locale. Ces types de rapports sont considérés comme "verticaux », parce qu'ils débordent les limites de la communauté. II en va ainsi des rapports qu'entretiennent les succursales de compagnies, d'organismes gouvernementaux ou d'associations (bureaux régionaux, sections locales, divisions, chapitres, etc.) avec leur siège social ainsi que des échanges de biens et services qui se font entre membres de communautés différentes. Conçu de cette façon, le tissu social communautaire ressemble à une "toile d'araignée " avec des fils (ou liens) horizontaux et verticaux (Williams, loc. cit., p. 90). La force de ce tissu social tient à sa construction et non aux valeurs ou aux sentiments qui animent le groupe, car nulle part dans les textes des auteurs américains il n'est question de 
" solidarité » au sens où l'entendent les sociologues européens et québécois.

De leur côté, Willems et Cuvillier (1970, p. 131) définissent I'horizontalité et la verticalité en fonction du statut social des gens. Dans leur perspective, la solidarité horizontale serait équivalente à celle qui unit une même classe ou strate sociale, alors que la solidarité verticale serait assimilable aux liens qui caractérisent les membres de diverses classes ou strates sociales à l'intérieur de certains groupes sociaux comme les églises et les partis politiques.

Enfin, l'horizontalité et la verticalité ont été abordées d'un point de vue générationnel par quelques auteurs, dont Bengtson et Schrader (1982, p. 118) et Zay (1984). Dans un article publié en 1984, Zay (p. 95) a décrit les différentes phases du développement d'une conscience de groupe parmi la population âgée. Ce processus commence par le rassemblement de personnes âgées au sein d'associations intragénérationnelles, passe par la prise de conscience des similitudes de condition de vie et aboutit au développement de la solidarité horizontale chez les aînés. Zay écrit :

Quel que soit le processus, l'apparition de la conscience de groupe crée un esprit de solidarité horizontale (" intragénérationnelle »), par opposition à un esprit de solidarité verticale (" intergénérationnelle »). Ces deux types de solidarités ne sont pas mutuellement exclusifs mais complémentaires. Si la gérontologie sociale encourage, à ce stade-ci de son développement, l'épanouissement de la solidarité "intragénérationnelle », c'est parce que celleci favorise l'autonomie par rapport à la solidarité « intergénérationnelle», qui tend à accélérer le phénomène de la parentification, c'est-à-dire la tendance à trouver refuge et soutien dans une figure parentale.

Pour Zay, le critère de différenciation entre solidarités horizontale et verticale est donc essentiellement générationnel. La dimension géographique de ces notions n'entre pas dans sa perspective, pas plus que l'aspect socioéconomique. Par ailleurs, la notion de solidarité à laquelle se réfère Zay se situe dans la tradition sociologique européenne d'utilisation de ce concept, car dans son optique la solidarité reste un rapport exclusif entre membres d'un groupe. 
Comme on le voit, l'horizontalité et la verticalité sont des concepts géométriques et géographiques qui, transposés à la sociologie, deviennent polysémiques par l'emploi qu'on en a fait. Dans tous les cas, l'horizontalité évoque les interrelations entre membres d'un même groupe, que celui-ci soit local, social ou générationnel, alors que la verticalité renvoie aux rapports intergroupes. Étant donné cette polysémie, il nous semble que l'emploi de concepts comme ceux de solidarité horizontale et verticale porte à confusion. Nous pensons qu'il serait plus clair et plus précis de parler de solidarité inter et intragénérationnelle. Quoi qu'il en soit, dans la suite de ce texte nous continuerons d'employer l'expression solidarité horizontale comme synonyme de solidarité reposant sur une base générationnelle.

\section{LES THÉORIES RELATIVES À LA SOLIDARITÉ HORIZONTALE OU INTRAGÉNÉRATIONNELLE}

II existe peu de théories permettant de décrire et d'expliquer la solidarité horizontale ou intragénérationnelle. Parmi celles-ci, mentionnons la théorie des générations de Mannheim, telle que reprise par quelques auteurs dont Attias-Donfut ainsi que Scott et Grasmick, la théorie de la stratification des âges de Riley et ses collaborateurs, de même que la théorie de la sous-culture des personnes âgées de Rose.

\section{La théorie des générations de Mannheim}

Comme nous l'avons vu, pour Mannheim (1952) les membres d'une même génération sont susceptibles de développer des affinités et d'adhérer à des valeurs communes parce qu'ils ont été influencés, au même âge, par des courants culturels analogues. Mais, pour qu'il en soit ainsi, il faut que les membres d'une même génération tissent des liens de coopération. S'ils s'ignorent mutuellement ou se font la lutte, il n'y aura pas émergence d'une conscience générationnelle ni développement d'une solidarité intragénérationnelle malgré le fait que les individus appartiennent à un même " espace générationnel », selon l'expression d'Attias-Donfut (1988). Autrement dit, dans cette perspective, la solidarité qui se forge entre les membres d'une génération est la conséquence de l'importance de l'âge dans une société en tant que critère de distribution des biens, rôles, fonctions, pouvoirs et privilèges, et en tant que facteur de différenciation culturelle. 
On doit à Mannheim le fait d'avoir énoncé les principes permettant de concevoir que des formes de solidarité puissent se forger en même temps que se constituent les générations, et cela est central dans la problématique de la solidarité horizontale. Quant à savoir comment il se fait qu'à certaines époques les jeunes, les adultes dans la force de l'âge ou les aînés deviennent conscients de leur appartenance à une même entité socioculturelle - leur génération - et qu'ils la constituent en un groupe concret pouvant devenir sujet de l'histoire, c'est une autre question à laquelle certains auteurs ont tenté de répondre. Toutefois, résumer leurs travaux nous écarterait du sujet de cet article. On peut consulter à ce propos Attias-Donfut (1988) et Mead (1979).

\section{La théorie de la stratification des âges}

Un texte sur la solidarité générationnelle ne saurait être complet sans évoquer la théorie de la stratification des âges (voir Riley, 1976, 1985 et 1994; Riley, Johnson et Foner, 1972). D'une part, les fondements de cette théorie rejoignent les propositions de Mannheim : le phénomène générationnel prédispose à la solidarité entre gens nés à la même époque, car ceux-ci peuvent avoir une culture et des valeurs communes. D'autre part, la succession des cohortes, c'est-à-dire le passage dans le temps de personnes nées à une même époque, entraîne la différenciation socioculturelle de leurs membres et suscite l'émergence d'une conscience générationnelle parce que le contexte historique alloue des rôles distincts à ceux qui appartiennent aux cohortes successives. Finalement, le processus de socialisation aux rôles singularise l'identité sociale des individus. Apparaissent alors des catégories de gens susceptibles de se regrouper et de tisser des liens, c'est-à-dire de devenir solidaires. Les clivages entre les générations favorisent donc l'établissement de liens de solidarité au sein de chacune d'elles.

\section{La théorie de la sous-culture des personnes âgées}

La théorie de la sous-culture des personnes âgées, qui a été formulée par A.M. Rose au début des années 1960, a été vérifiée quelquefois par la suite (Rose, 1965; voir aussi Crandall, 1991, p. 104-106). Cette théorie est centrale dans la problématique de la solidarité horizontale, et c'est pourquoi nous lui accordons un traitement privilégié. 
La notion de sous-culture est issue de l'anthropologie sociale des années 1950 et 1960 . Cette notion désignait alors les normes, valeurs et attitudes des groupes marginaux de la société, en particulier celles des paurres et des jeunes délinquants (Willems et Cuvillier, 1970). De façon plus précise, une sousculture ou subculture est une «Culture particulière à un groupe faisant partie d'une société plus vaste avec laquelle il partage certaines caractéristiques » (Zay, 1981, p. 527). Quant à la culture, elle réunit en un tout cohérent les valeurs auxquelles adhèrent les membres d'une société ainsi que les normes de comportement (modèles culturels) auxquelles ils sont censés se conformer (Rocher, 1969). La culture est donc un univers symbolique qui donne une signification aux gestes posés par les gens.

Principes fondamentaux de la théorie de la sous-culture des personnes âgées

1. Il existe une pluralité de sous-cultures : d'âge, de sexe, de classe sociale, de milieu socioprofessionnel, etc. Parmi ces sous-cultures, celle des groupes d'âge est prédominante. Dès lors, les membres d'un même groupe d'âge sont prédisposés à prendre conscience de leur situation et à s'identifier à leur pairs. C'est ce qu'on appelle la « conscience générationnelle » (Aging Group Consciousness; Aging Group Identification).

2. Les personnes âgées forment un sous-groupe de la société qui a des caractéristiques spécifiques et sa propre culture: absence de travail professionnel et d'obligations familiales; lieux de résidence communs; valeurs et centres d'intérêt analogues, etc.

3. Cependant, toutes les personnes âgées n'adhèrent pas aussi fortement aux valeurs de leur sous-culture d'âge. Les gens âgés les plus favorisés économiquement s'identifient moins à leurs pairs. II en va de même de ceux qui ont une santé défaillante, de ceux qui se replient sur leur vie familiale ou, encore, de ceux qui sont en bonne santé et qui ne se perçoivent pas comme vieux. Par contre, ceux qui vivent avec d'autres personnes âgées sont davantage susceptibles de souscrire à de telles valeurs. 
4. Les aînés qui interagissent fréquemment avec leurs pairs et qui souscrivent aux valeurs de leur sous-culture d'âge devraient être mieux intégrés socialement et avoir une meilleure estime d'eux-mêmes.

5. Les aînés qui partagent les valeurs de leur sous-culture devraient être actifs sur le plan politique et défendre les intérêts de leurs pairs.

Cette théorie est celle qui étaie le mieux l'idée de solidarité horizontale. En effet, si l'«espace générationnel » des gens âgés constitue une sous-culture, ces gens devraient être naturellement solidaires, entretenir des échanges privilégiés et s'entraider, dimensions fondamentales de la solidarité intragénérationnelle.

La principale critique formulée à l'endroit de cette théorie est que les aînés « constitueraient plus un agrégat ou une catégorie qu'une subculture » du fait qu'ils "subissent l'action des mêmes forces économiques » (Zay, 1981, p. 528). Dès lors, ils n'auraient pas « d'intérêts communs propres à favoriser la naissance d'une conscience de groupe » (ibid.). Quoi qu'il en soit, les hypothèses qui découlent de la théorie de la sous-culture des personnes âgées ont été vérifiées empiriquement, comme on le verra dans la section suivante.

\section{LES RECHERCHES EMPIRIQUES SUR LA SOLIDARITÉ HORIZONTALE CHEZ LES PERSONNES ÂGÉES}

\section{Les indicateurs de la solidarité horizontale}

Divers indicateurs ont été utilisés pour mesurer l'ampleur de la solidarité horizontale chez les aînés. D'une part, il y a la conscience générationnelle des gens âgés, leur identification à ce groupe d'individus et leur politisation. D'autre part, il y a les indicateurs de participation sociale des personnes âgées: l'importance des groupes d'aînés et le fait de privilégier l'appartenance à ces groupes plutôt qu'aux associations multigénérationnelles. Enfin, il y a les indicateurs de l'entraide chez les gens âgés, soit les échanges de services, notamment dans les associations de gens âgés. Aucune donnée considérée isolément ne permet de conclure en l'existence ou en l'inexistence de la solidarité horizontale chez les aînés, mais l'ensemble des informations colligées montre que ce concept a une emprise sur la réalité. 


\section{La conscience générationnelle, l'identification au groupe des aînés et leur politisation}

Quelques études ont été effectuées pour savoir dans quelle mesure les aînés s'identifient à leurs pairs et s'ils sont conscients d'appartenir à une génération ayant une certaine cohésion. Dans une de ces études, effectuée auprès de 221 personnes de 18 ans et plus de Minneapolis City aux États-Unis, on a constaté qu'entre l'âge de 18 ans et de 32 ans il y a un rapide déclin de la proportion de gens disant appartenir à la jeune génération, mais entre 32 et 36 ans ce déclin est beaucoup plus lent (Scott et Grasmick, 1979). Puis, de façon surprenante, entre 36 et 45 ans, il y a augmentation de la proportion de gens qui s'identifient à la jeune génération. Comme si les individus qui sont au tournant de la quarantaine refusaient d'assumer leur vieillissement. Après quoi, cette proportion diminue rapidement pour devenir presque nulle au-delà de 50 ans. I semble donc que la «crise du milieu de la vie " soit une réalité qui amène beaucoup de personnes ( $40 \%$ des individus dans la quarantaine) à nier le fait qu'ils vieillissent.

De plus, environ la moitié des individus qui s'identifient à la jeune génération et à la plus âgée considèrent que les membres d'une même génération ont une forte cohésion (" usually stick together »). Aussi, des proportions identiques de gens s'identifiant à la jeune génération et à la plus âgée croient que les membres des différentes générations s'entendent bien dans la société ( usually get along well »), donc ne perçoivent pas d'antagonismes entre les groupes d'âge. Ce n'est pas tout. Une plus forte proportion de gens s'identifiant à la jeune génération pensent que les politiques gouvernementales qui visent à aider une génération nuisent à l'autre groupe d'âge (" usually or sometimes are not good for the other $)$ ), laissant croire à une plus grande politisation des jeunes par rapport aux plus âgés.

Les auteurs concluent que la conscience générationnelle est une réalité intrinsèque de la société américaine, que les limites («boundaries ») des différentes générations sont clairement établies, que la majorité des gens préfèrent interagir avec les membres de leur génération et que les générations sont politisées. Leurs résultats confirment les théories voulant que les générations aient tendance à se refermer sur elles-mêmes. Les auteurs se demandent toutefois si ces phénomènes résultent de 
contraintes structurelles (l'isolement relatif des diverses générations dans la société) ou d'une intention consciente des acteurs. Le cas échéant, la solidarité intragénérationnelle (les auteurs n'emploient pas cette expression), si tant est qu'elle existe, serait plus un effet de l'organisation sociale que l'expression de la volonté des membres des divers groupes d'âge.

D'autres études du genre ont été effectuées durant les années 1970 aux États-Unis. Dans son étude, Lau (1989; $n=2705$ et $n=2248$; échantillons représentatifs de l'électorat américain) a demandé aux gens s'ils se sentaient près de certains groupes («Feel close to...»), étant conscient de la signification quelque peu ambiguë que pouvaient avoir les réponses à une telle question. L'auteur a considéré comme jeunes les individus de 17 à 29 ans et comme âgés, ceux de 60 ans et plus. II a constaté que les mêmes pourcentages de jeunes et de plus âgés (68\%) s'identifiaient respectivement à la jeune génération et à celle des aînés, corroborant ainsi la recherche de Scott et Grasmick. Ensuite, Lau a observé que l'âge et la scolarité étaient les principaux prédicteurs de l'identification à la jeune génération, tandis que le fait d'être retraité et le sexe (féminin) étaient les variables qui expliquaient le mieux l'identification à celle des aînés.

Ces résultats suggèrent que le fait d'être jeune a plus de signification pour les jeunes que le fait d'être vieux pour les gens âgés. Car la jeunesse est clairement identifiée dans les sociétés occidentales, et il est valorisant d'être jeune. Par conséquent, la sous-culture des jeunes serait plus nettement affirmée que celle des aînés, cette sous-culture étant encore en émergence. Dans les sociétés occidentales, l'appartenance au groupe des aînés se définit par l'occupation, notamment par le fait d'être retraité. C'est probablement ce facteur qui constitue le fondement de l'identité sociale des gens âgés, et non l'âge proprement dit (" $\grave{A}$ quel âge devient-on vieux? »). Le statut de retraité favoriserait donc le développement de liens de solidarité, un peu comme la place occupée dans les rapports de production est à l'origine de la conscience de classe.

Une étude a été menée pour vérifier directement les hypothèses découlant de la théorie de la sous-culture des personnes âgées (Longino, McCelland et Peterson, 1980; voir aussi Crandall, 1991). Pour ce faire, les auteurs ont comparé les résidants de huit communautés de retraités à un échantillon de personnes âgées demeurant à domicile dans un environnement 
multigénérationnel. Les résultats de cette étude indiquent que, par rapport aux aînés qui vivent en milieu multigénérationnel, ceux qui demeurent dans des communautés de retraités sont mieux intégrés socialement; ils ont davantage d'interactions avec les autres personnes âgées; ils ont une meilleure image des membres de ce groupe d'âge et d'eux-mêmes; ils s'ennuient moins et se sentent plus utiles. Mais, contrairement aux hypothèses de la théorie, ceux qui vivent dans des communautés de retraités ne sont pas plus politisés; ils ont tendance à être "retraitistes » plutôt qu' « activistes » sur ce plan.

Cette recherche, qui corrobore en partie celles de Carp (1966) et de Rosow (1967 et 1974), montre qu'une sous-culture du troisième âge émerge là où les personnes âgées sont concentrées, lieux où se développent « naturellement » des formes de solidarité. Est-ce à dire que la solidarité horizontale chez les aînés est essentiellement conjoncturelle, pour ne pas dire accidentelle, que cette solidarité émerge dans des circonstances particulières et qu'elle n'est pas un trait caractéristique de l'existence collective des gens âgés? C'est ce qui se dégage des travaux évoqués ici, mais il ne faut pas sauter trop vite aux conclusions.

\section{La participation sociale des personnes âgées}

La participation sociale des personnes âgées est un autre indicateur de l'existence d'une solidarité intragénérationnelle chez ces personnes. En effet, si les aînés sont solidaires, ils auront tendance à se regrouper dans des associations de gens âgés, à privilégier l'appartenance à de telles associations et à considérer comme moins important le fait d'être membre d'organisations multigénérationnelles. Les données recueillies à ce sujet corroborent ces assertions.

Ainsi, comme le montre le tableau 1, la majorité des aînés québécois francophones qui appartiennent à des groupes communautaires font partie d'associations de gens âgés. Cette proportion varie entre $61 \%$ et $95 \%$ selon les régions du Québec étudiées par Delisle (1995). Seule exception: les anglophones de l'Estrie où les groupes de l'âge d'or sont moins fréquentés, pour des raisons qui restent à établir. De plus, l'attachement aux groupes d'aînés n'est pas l'apanage des Québécois francophones âgés, car ce phénomène a été observé ailleurs en Amérique du 
TABleau 1

Participation sociale nominale (\%)

Régions et groupes linguistiques

\begin{tabular}{|c|c|c|c|c|c|c|c|c|c|c|}
\hline & Mauricie & & Estrie & & Gaspésie & Portneuf & $\begin{array}{l}\text { Lotbinière } \\
\text { Alli-Äges }\end{array}$ & Saguenay & $\begin{array}{l}\text { Lac- } \\
\text { Saint-Jean }\end{array}$ & $\begin{array}{l}\text { Ensemble } \\
\text { du Québec }\end{array}$ \\
\hline $\begin{array}{l}\text { Participation } \\
\text { sociale }\end{array}$ & & Anglos & Francos & Tous & & & & & & \\
\hline Oui & 62,4 & 61,0 & 43,4 & 50,6 & 42,2 & 54,4 & 66,4 & 53,0 & 52,1 & 36,0 \\
\hline $\begin{array}{l}\text { Non et sans } \\
\text { réponse }\end{array}$ & 37,6 & 39,0 & 56,6 & 49,4 & 57,8 & 45,6 & 33,6 & 47,0 & 47.9 & 64,0 \\
\hline \multicolumn{11}{|l|}{ Groupes d'aînés } \\
\hline $\begin{array}{l}\text { Groupes d'aînés/ } \\
n \text { néchantillon }\end{array}$ & 41,7 & 26,5 & 34,3 & 31,1 & 37,4 & 45,2 & 62,9 & $-"$ & - & - \\
\hline $\begin{array}{l}\text { Groupes d'ainés/ } \\
n \text { adhérant à des } \\
\text { groupes }\end{array}$ & 66,9 & 43,4 & 79,1 & 61,5 & 89,3 & 83,1 & 94,7 & - & - & - \\
\hline $\begin{array}{l}n \text { adhérant à des } \\
\text { groupes }\end{array}$ & 181 & 83 & 86 & 169 & 121 & 166 & 245 & 214 & 394 & 245 \\
\hline néchantillon & 290 & 136 & 198 & 334 & 289 & 305 & 369 & 404 & 756 & 680 \\
\hline
\end{tabular}

* - : donnée non disponible.

Source : Delisle, 1995, p. 73. Voir œ document pour les précisions méthodologiques. 
Nord et en Europe (Attias-Donfut et Rozenkier, 1983; Crandall, 1991). Les membres d'une même génération ont sans doute plus de choses en commun que ceux de générations différentes, ce qui crée une situation favorable à l'émergence de la conscience et de la solidarité intragénérationnelles (Gallagher, 1994, p. 33-34; Scott et Grasmick, 1979, p. 198) ${ }^{2}$.

Chez les Québécois âgés, l'attachement aux groupes d'aînés est très fort. Ainsi, la Fédération de l'âge d'or du Québec a demandé au Groupe Léger et Léger d'effectuer un sondage auprès de ses membres. Ce sondage a montré que $88,4 \%$ des répondants jugeaient très important et assez important d'être membre de la FADOQ, alors que $48 \%$ d'entre eux trouvaient très important ou assez important d'être membre d'un autre club ou association (FADOQ, 1993 et Magazine FADOQ, 1993, vol. 2, $n^{\circ} 5$, p. 33-34) ${ }^{3}$. La principale raison donnée par les personnes qui trouvaient important d'être membre de la FADOQ était le fait de pouvoir rencontrer des personnes de leur âge (39,6\% de l'ensemble des répondants), tandis que la principale raison donnée par les individus qui considéraient important d'être membre d'un autre club ou association était le fait de pouvoir rencontrer des personnes d'autres milieux $(24,2 \%$ de l'ensemble des répondants). Incidemment, $83,2 \%$ des aînés interviewés ont affirmé qu'ils trouvaient très important ou assez important de pouvoir rencontrer des personnes de leur âge et de parler avec elles.

Ces données sont révélatrices de l'identification des aînés à leur génération, composante fondamentale de la sous-culture du troisième âge et de la conscience intragénérationnelle. Elles confirment ce qu'on peut observer dans les congrès de la FADOQ : ses membres s'identifient à leurs pairs, car à la FADOQ le fait d'être âgé n'est pas gênant; c'est même une source de prestige. Il en va sans doute ainsi dans les autres groupes d'aînés, comme le suggère l'étude de Lachance (1990). Sa recherche a montré que les médias de l'âge d'or projetaient une image positive et

2. L'étude de Gallagher a été effectuée auprès de 324 hommes et femmes de 25 à 94 ans demeurant à Springfield, Mass.

3. Sondage réalisé en février 1993 par le Groupe Léger \& Léger auprès d'un échantillon représentatif de 1002 membres de clubs de l'âge d'or affiliés à la Fédération. 
valorisante des aînés, image qui contredit les stéréotypes négatifs souvent véhiculés à leur endroit.

\section{L'entraide chez les personnes âgées en général et chez celles qui fréquentent les groupes d'aînés}

Qu'en est-il maintenant de l'entraide chez les aînés? Cette question sera abordée sous les aspects suivants: 1) l'effet de la corésidence dans un même lieu; 2) le soutien mutuel; 3) l'effet du regroupement en associations; 4) les rapports entre les aînés et les adultes plus jeunes, et 5) l'organisation de l'entraide dans les groupes. Premièrement, les gens âgés qui sont entourés de membres de leur génération s'entraident plus que les personnes âgées résidant dans un environnement multigénérationnel. Cette constatation est ressortie de plusieurs études et renvoie presque à une évidence : on a tendance à échanger des services avec ceux qu'on côtoie immédiatement et ce, nonobstant leur âge (Bélanger et Delisle, 1981; Carp, 1966 et 1976; Delisle, 1994; Delisle, Boucher et Roy, 1993; Rosow, 1967 et 1974).

Deuxièmement, l'aide donnée par des aînés à d'autres aînés est considérable, ce qu'ont montré plusieurs recherches (Corin [dir.], 1983; Delisle, 1994; Delisle, Boucher et Roy, 1993; Delisle et Ouellet, 1996; Garant et Bolduc, 1990; Renaud [dir.], 1987; Roy, Vézina et Cliche, 1996). Ainsi, $46 \%$ des personnes de 65 ans et plus demeurant sur le territoire du CLSC La Source (Charlesbourg) et $37 \%$ de leurs homologues du comté de Portneuf ont donné de l'aide à une autre personne de 65 ans et plus au cours des trois mois ayant précédé l'étude (automne 1995 à La Source et automne 1991 dans Portneuf). Réciproquement, $21,4 \%$ et $24,3 \%$ des répondants de chacune des études ont reçu de l'aide d'un membre de ce groupe d'âge pendant la période considérée (pour La Source, voir Roy, Vézina et Cliche, 1996, p. 71; pour Portneuf, voir Delisle, Boucher et Roy, 1993, p. 65). Nous ne connaissons pas de données équivalentes pour l'ensemble de la population du Québec et du Canada. Cependant, on sait que les conjoints des personnes âgées, leurs frères et sœurs, leurs amis, leurs voisins et les membres de leur communauté locale, qui sont souvent des aînés, fournissent beaucoup de soutien à leurs proches âgés (ibid.). Signalons aussi que l'aide reçue par les gens âgés varie en fonction du sexe et du statut marital. Les femmes - et les veuves en particulier - échangent avec leurs proches (de tout âge) plus de services que les hommes et les veufs (Gallagher, 1994; Kohen, 1983; Stryckman, 
1982). Enfin, l'aide donnée par les personnes âgées n'est pas réservée aux aînés. Les études réalisées dans Portneuf et dans Charlesbourg, entre autres, indiquent que les personnes âgées aident beaucoup les membres des générations plus jeunes.

Troisièmement, le regroupement des aînés en associations favorise l'entraide chez ces personnes. Plusieurs résultats de recherche fondent cette assertion. D'une part, selon Gallagher (1994), les aînés ont plus tendance à faire partie de groupes soutenant les membres de leur génération que les adultes plus jeunes. On peut donc croire que les gens âgés sont conscients de la nécessité d'aider leurs semblables qui sont dans le besoin. D'autre part, plusieurs aînés membres de groupes sociaux, et notamment de groupes d'aînés, sont portés à faire du bénévolat auprès de leurs pairs dans le cadre des activités de ces organisations. Ainsi, à La Source, $35 \%$ des gens âgés membres d'associations le font (Roy, Vézina et Cliche, 1996, p. 72). Dans Portneuf, cette proportion est de $22 \%$ (Delisle, Boucher et Roy, 1993, p. 69). II en va de même dans le Bas-Saint-Laurent, où $65 \%$ des gens âgés appartenant à un «club santé » donnent de l'aide à leurs collègues et où $49 \%$ de ces aînés en reçoivent de la part de personnes âgées qu'ils côtoient (Bélanger, Gilbert et Pelletier, 1988, p. 28). Dans Lotbinière, $49 \%$ des gens de 65 ans et plus membres de groupes d'aînés ont rendu service à un de leurs pairs pendant le trimestre ayant précédé l'étude, et $51 \%$ des aînés membres de groupes du troisième âge ont reçu du soutien d'un de leurs collègues ${ }^{4}$. Sur la Rive-Sud de Québec (MRC Desjardins et Chutes-de-la-Chaudière), ces pourcentages sont respectivement de $53,8 \%$ et $46,6 \%$ (Delisle et Ouellet, 1996, p. 4). L'aide dont il est question ici prend diverses formes : courses, entretien domestique, transport, visites à l'hôpital, réconfort, conseils, orientation vers les services disponibles, etc. Cependant, l'aide fournie par des individus extérieurs à la famille touche rarement au domaine du caregiving, c'est-à-dire à celui des soins et services personnels. Cela corrobore d'autres études et suscite des doutes quant à la possibilité que cette aide puisse contribuer significativement au maintien à domicile des aînés en perte d'autonomie (Béland, 1984; Crohan et Antonucci, 1989;

4. Compilations effectuées par Gale E West et Marc-André Delisle à partir des données de Simard, Delisle, Drouin et Larouche (1992). Pour des précisions méthodologiques, voir West, Delisle, Simard et Drouin (1996). 
Garant et Bolduc, 1990; Johnson et Troll, 1994). Mais cette question est connexe à notre propos et nous ne pouvons pas en traiter ici. Quoi qu'il en soit, il semble que les échanges soient bilatéraux, car la plupart des gens âgés qui rendent service à leurs homologues en reçoivent de ceux-ci. En effet, comme l'indique le tableau 2, la relation entre l'aide donnée et l'aide reçue est statistiquement significative. II faut quand même préciser que les données disponibles ne permettent pas de savoir si la personne qui a rendu service à quelqu'un a reçu de l'aide de ce même individu.

\section{TABLEAU 2}

Services donnés par des personnes de 65 ans et plus

\begin{tabular}{lcc}
\hline & Lotbinière & Rive-Sud de Québec \\
\hline Ont donné et reçu & 28,3 & 37,6 \\
Ont donné seulement & 20,7 & 16,3 \\
Ont reçu seulement & 17,4 & 9,0 \\
N'ont ni donné ni reçu & 33,6 & 37,1 \\
$n$ & 418 & 358 \\
& $\chi^{2}=15,3 p<0,001$ & $\chi^{2}=55,5 \quad p<0,0001$ \\
\hline
\end{tabular}

* Services donnés par des membres d'organisations d'aînés à leurs homologues et services reçus de leur part. Personnes de 65 ans et plus.

Pourcentages calculés sur les échantillons totaux. Comté de Lotbinière (1992) et MRC Desjardins et Chutes-de-la-Chaudière (1996).

Sources : - Lotbinière Données non publiées issues de l'étude de Simard, Delisle, Drouin et Larouche (1992). Voir West, Delisle, Simard et Drouin (1996).

- Rive-Sud Données non publiées issues de l'étude de Delisle et Ouellet (1996).

Quatrièmement, les associations volontaires de gens âgés fonctionnent de façon que les divers types de relations sociales (avec la famille, les amis et les voisins) puissent s'harmoniser, ce qui renforce leur réseau de soutien (Breytspraak, Halpert et Olson, 1985, p. 285). Les analyses secondaires bivariées effectuées à partir des études réalisées dans les comtés de Portneuf et de Lotbinière corroborent cette assertion. Dans Portneuf, le nombre de groupes auxquels appartient la personne âgée et la fréquence de sa participation aux activités des associations sont significativement $(p<0,05)$ et positivement corrélés : aux 
services rendus à l'entourage $(r=0,28 \text { et } r=0,29)^{5}$; aux services reçus de l'entourage $(r=0,15$ et $r=0,17)$; aux services rendus à des gens de 65 ans et plus $(r=0,28 \text { dans les deux cas })^{6}$, et aux contacts avec la famille $(r=0,36$ et $r=0,32)$. Dans Lotbinière, la fréquence de la participation aux activités des groupes (composés principalement d'aînés) est corrélée significativement par rapport aux contacts avec la famille $(r=0,11)$ et avec les non-parents ${ }^{7}(r=0,23)$. La participation sociale fournit donc la possibilité aux personnes âgées d'échanger des services avec leurs pairs sans négliger leurs proches plus jeunes.

Enfin, l'Association des retraités de l'enseignement du Québec (AREQ) a effectué un sondage auprès de ses membres pour connaître leur profil socio-démographique et économique ainsi que leurs conditions de vie, besoins et aspirations (Toussaint, 1985). Cette étude a révélé que les besoins perçus et les attentes envers les organismes sans but lucratif se situaient dans les domaines suivants : la possibilité de rencontrer des amis, de parler à quelqu'un, d'avoir des activités de loisir, d'avoir de la visite, d'être aidé dans l'administration de ses affaires et pour l'entretien domestique. $\dot{A}$ la suite de ce rapport, un sondage maison a été effectué pour savoir ce qui se faisait localement en matière d'entraide (Toussaint, 1988-1989). Cette enquête a montré que la plupart des comités des régions et secteurs avaient pris des initiatives pour favoriser l'entraide entre les membres de l'AREQ. II s'agissait notamment de covoiturage, d'information sur les activités de l'Association et sur les services sociosanitaires, de services de dépannage ainsi que de parrainage entre un membre dans le besoin et un membre bénévole. Ce sondage a également permis de constater que l'entraide était organisée de façon non systématique, et que cela rendait ardue la tâche de ceux qui voulaient en faire la promotion.

La situation observée à l'AREQ est un " cas de figure " qui reflète probablement assez bien ce qui se passe dans les associations d'aînés. L'entraide entre les membres est perçue comme une dimension importante de la vie de ces groupes, mais ce

5. Soit le conjoint, les enfants ainsi que des personnes de moins 65 ans et de 65 ans et plus autres que le conjoint et les enfants.

6. Cette catégorie d'individus n'inclut pas le conjoint.

7. Amis, voisins, membres de groupes. 
n'est point leur raison d'être. De ce fait, ils ne sont pas portés à l'organiser de façon systématique.

\section{CONCLUSION}

En conclusion, nous tenterons de répondre précisément aux interrogations soulevées dans l'introduction. La première de ces questions portait sur les assises théoriques du concept de solidarité horizontale. La documentation consultée montre que c'est dans la première moitié du vingtième siècle que des théories pouvant expliquer ce phénomène ont été formulées. Les hypothèses découlant de la théorie des générations de Mannheim, de celle de la stratification des âges et de celle de la sous-culture des personnes âgées suggèrent que les générations peuvent se comporter comme des "groupes concrets " ayant une assez forte cohésion, dans un contexte sociohistorique donné. Ce phénomène a pour cause la marginalité relative des différentes générations dans la société, marginalité qui incite les membres des diverses générations à accroître leurs interactions et à se définir de plus en plus en fonction de leurs collatéraux d'âge. Car la position marginale d'un groupe définit un « rapport cognitif au monde ", rapport qui établit un clivage entre un " nous », les semblables, et un "eux", les autres (Howard, 1994). Cette situation peut entraîner le développement d'une conscience générationnelle et l'établissement de liens de solidarité entre les membres de chacune des générations.

Quant à la deuxième question, elle portait sur l'importance de la solidarité horizontale chez les aînés. Les données rassemblées indiquent que la conscience générationnelle existe bel et bien chez les aînés, et que la plupart d'entre eux s'identifient à leurs pairs. Par contre, les études consultées ne révèlent pas un haut degré de politisation chez les aînés actuels. Mais qu'en sera-t-il dans l'avenir? En ce qui a trait aux données sur la participation sociale des personnes âgées, elles sont révélatrices de l'existence d'une sphère et d'une sous-culture du troisième âge. En effet, les aînés ont tendance à se regrouper dans des associations de gens âgés et à préférer l'appartenance à de telles associations, ce qui favorise l'établissement d'échanges privilégiés entre leurs membres. Toutefois, ces échanges se font de manière informelle ou à l'initiative de certains leaders, car les groupes d'aînés ne sont pas en mesure ou ne se sentent pas tenus de les organiser sur une base institutionnelle. 
Au terme de cette démarche, nous retiendrons ceci. La solidarité horizontale ou intragénérationnelle est une réalité chez les personnes âgées. Cependant, cette réalité est contextuelle. Autrement dit, il y a des contextes et des sphères dans lesquels cette solidarité se manifeste, mais elle n'apparaît pas dans tous les contextes et dans toutes les sphères où évoluent les personnes âgées. C'est que la marginalité des aînés est toute relative dans les sociétés occidentales contemporaines, car la sphère de ces personnes n'est pas totalement dissociée ni totalement coupée de celle des adultes plus jeunes. Reste que cette dissociation et cette dislocation sont assez importantes pour obliger les aînés à développer des formes de solidarité qui leur permettent de mieux satisfaire certains de leurs besoins. Mais la documentation examinée ne fournit guère plus d'informations sur ce sujet.

Or, la problématique de la solidarité horizontale chez les gens âgés mérite davantage d'approfondissement, en raison de son importance stratégique au regard des politiques sociales relatives à la vieillesse. Dès lors, des travaux de recherche devraient être entrepris sur ce phénomène, travaux qui auraient pour mission d'examiner de façon systémique les éléments des sphères (temps, espace, personnes) où se vit cette solidarité, de même que les facteurs constitutifs des contextes favorables à l'émergence de la solidarité horizontale (sur la morphologie des sphères et des contextes, voir Delisle, 1987a et b). II importe en effet de savoir dans quel environnement apparaît et se développe ce type de solidarité chez les personnes âgées. De même, il est nécessaire d'identifier les facteurs qui facilitent ou entravent son développement. Seules de telles études sont susceptibles de produire les éléments de connaissance essentiels à la conception de modes d'intervention pouvant tirer profit de la solidarité horizontale chez les aînés.

Marc-André DELISLE

Chargé de cours Département de sociologie Université Laval 


\section{Références bibliographiques}

ATTIAS-DONFUT, C. (1993). "Générations et repères culturels 》, Loisir et Société, vol. 15, n², p. 419-435.

ATTIAS-DONFUT, C. (1991). Générations et âges de la vie, Paris, PUF. Coll. Que sais-je?, $n^{\circ} 2570$.

ATTIAS-DONFUT, C. (1988). Sociologie des générations : L'empreinte du temps, Paris, PUF.

ATTIAS-DONFUT, C et A. ROZENKIER (1983). "Des clubs du $3^{\circ}$ âge aux mouvements associatifs: une dynamisation sociale des personnes âgées? ", Gérontologie et société, vol. 26, p. 89100.

BÉLAND, F. (1984). «L'effet de la corésidence sur les désirs d'hébergement des personnes âgées », Recherches sociographiques, vol. XXV, n², p. 267-283.

BÉLANGER, D., M. GILBERT et J. PELLETIER (1988). Évaluation d'impact des activités collectives de prévention primaire, Rivièredu-Loup, Québec, Département de santé communautaire du Centre hospitalier régional du Grand-Portage.

BÉLANGER, L. et M.-A. DELISLE (1981). Les habitations à loyer modique pour personnes âgées: effets psychologiques et sociaux de l'entrée en HLM et du refus de la demande d'admission, Québec, Laboratoire de gérontologie sociale de l'Université Laval.

BENGTSON, V. L. et S.S. SCHRAdER (1982). "Parent-Child Relations », dans D.J. Mangen et W.A. Peterson, Research Instruments in Social Gerontology, vol. 1, Minneapolis, University of Minnesota Press, p. 115-185.

BOUDON, R (dir.) (1989). Dictionnaire de sociologie, Paris, Larousse.

BRÉMOND, J. et A. GELEDAN (1981). Dictionnaire économique et social, Paris, Hatier.

BREYTSPRAAK, L.M., B.P. HALPERT et P.G. OLSON (1985). "The Voluntary Organization as a Support System in the Aging Process ", dans W.A. Peterson et J. Quadagno (dir.), Social Bonds in Later Life, Aging and Interdependance, Beverly Hills, Sage Publ., p. 273-286.

CARP, F. (1966). A Future for the Aged Victoria Plaza and Its Residents, Austin and London, University of Texas Press.

CARP, F. (1976). " Housing and Living Environments of Older People », dans RH. Binstock et E. Shanas (dir.)., Handbook of Aging and the Social Sciences, New York, Van Nostrand Reinhold, p. 244-269. 
CORIN, E.E. (dir.) (1983). Le fonctionnement des systèmes de support naturel, vol. 1, Québec, Laboratoire de gérontologie sociale de l'Université Laval.

CRANDALL, R.C. (1991). Gerontology, A Behavioral Science Approach, $2^{\mathrm{e}}$ éd., New York, McGraw-Hill.

CROHAN, S.E. et T.C. ANTONUCCI (1989). "Friends as Source of Social Support in Old Age », dans R.G. Adams et R. Blieszner (dir.), Older Adult Friendship, Structure and Process, Newbury Park, CA, Sage Publication, p. 129-146.

DELISLE, M.-A. (1996). Aspects démographiques, économiques et sociologiques du vieillissement, Sainte-Foy, Éditions La Liberté.

DELISLE, M.-A. (1995). Vieillir dans les régions, Etude comparative des conditions et du mode de vie des aînés demeurant hors des grands centres urbains du Québec, Sainte-Foy, Éditions La Liberté.

DELISLE, M.-A. (1994). " La solidarité horizontale chez les personnes âgées du comté de Portneuf », Le Gérontophile, vol. $16, n^{\circ} 4$, p. $15-23$.

DELISLE, M.-A. (1987a). La République du silence: Solitude et vieillissement, Québec, Laboratoire de recherches sociologiques de l'Université Laval. Coll. Rapports de recherche, $\mathrm{n}^{\circ} 25$.

DELISLE, M.-A. (1987b). "La demande de services socio-sanitaires publics de la part des personnes âgées : éléments de problématique et directions de recherche », dans G. Leclerc et collab., Les conditions de l'autonomie individuelle et collective de la personne âgée, Cahiers de l'ACFAS, $n^{\circ} 56$, Montréal, Association canadienne-française pour l'avancement des sciences, p. 73-105.

DELISLE, M.-A., N. BOUCHER et J. ROY (1993). " ...Pour s'entraider ». Étude portant sur la solidarité horizontale chez les personnes âgées du comté de Portneuf. Québec, Centre de recherche sur les services communautaires de l'Université Laval.

DELISLE, M.-A. et H. OUELLET (1996). Recherche sur la participation sociale et le recours aux services, Principaux faits saillants, Lévis, Maison des aînés de Lévis.

DURKHEIM, E. (1978). De la division du travail social, Paris, PUF.

FADOQ (Fédération de l'âge d'or du Québec) (1993). Évaluation des perceptions des membres de la FADOQ à l'égard des activités sociales en général, leur satisfaction à l'égard de la FADOQ, leur implication dans d'autres clubs et associations et 
leurs attentes et besoins, Montréal, Fédération de l'âge d'or du Québec/Groupe Léger \& Léger.

FRANCE, H. et C. MCDOWELL (1982). " L'entraide chez les personnes âgées: modèle de counselling par les pairs », Santé mentale au Canada, vol. 30, $\mathrm{n}^{\circ} 3$, p. 15-17.

GALLAGHER, S.K. (1994). Older People Giving Care, Helping Family and Community, Westport, Conn., Auburn House.

GARANT, L. et M. BOLDUC (1990). L'aide par les proches: mythes et réalités, Revue de littérature et réflexions sur les personnes âgées en perte d'autonomie, leurs aidants et aidantes naturels et le lien avec les services formels, Coll. "Études et analyses " $n^{\circ} 8$, Québec, ministère de la Santé et des Services sociaux, Direction de l'évaluation.

GIRARDET, R. (1983). "Du concept de génération », Revue d'histoire moderne et contemporaine, vol. XXX, p. 257-270.

GUILLEMARD, A.-M. (1989). "Solidarité (politique de)", dans R. Boudon (dir.), Dictionnaire de sociologie, Paris, Larousse, p. $186-187$.

GUIMOND, S. (1994). "Les groupes sociaux », chap. 12 dans Robert J. Vallerand (dir.), Les fondements de la psychologie sociale, Boucherville, Gaëtan Morin éditeur, p. 655-705.

HOWARD, J.A. (1994). "A Social Cognitive Conception of Social Structure », Social Psychology Quarterly, vol. 57, n 3, p. 210227.

JOHNSON, C.L. et L.E. TROLL (1994). "Constraints and Facilitators to Friendships in Late Life », The Gerontologist, vol. 34, $n^{\circ} 1$, p. $79-87$.

KOHEN, J.A. (1983). «Old But Not Alone: Informal Social Supports Among The Elderly by Marital Status and Sex », The Gerontologist, vol. 23, n० 1, p. 57-63.

LACHANCE, G. (1990). Nouvelles images de la vieillesse, Une étude de la presse âgée au Québec, Documents de recherche $n^{\circ} 22$, Québec, Institut québécois de recherche sur la culture.

LAU, R R (1989). «Individual and Contextual Influences on Group Identification», Social Psychology Quarterly, vol. 52, $n^{\circ} 3$, p. 220-231.

LONGINO, C.F., MCCELLAND, F.A. et W.A. PETERSON (1980). «The Aged Subculture Hypothesis: Social Integration, Gerontophilia and Self-Conception », Journal of Gerontology, vol. 35, $n^{\circ} 5$, p. $758-767$.

MANNHEIM, K. (1952). "The Problem of Generation 》, Essays on the Sociology of Knowledge, New York, Oxford University 
Press, p. 276-320. En français: Le problème des générations, Paris, Nathan, 1990.

MEAD, M. (1979). Le fossé des générations: Les années 70, Paris, Denoël/Gonthier.

MUDRACK, P.E. (1989). « Defining Group Cohesiveness, A Legacy of Confusion? », Small Group Behavior, vol. 20, n 1, p. 37-49.

RENAUD, M. (dir.) (1987). Les solutions qu'apportent les Québécois à leurs problèmes sociaux et sanitaires, Rapport présenté à la Commission d'enquête sur les services de santé et les services sociaux, Québec, Les Publications du Québec.

RIESSMAN, F. (1982). Self-Help and the Elderly, Considerations for Practice and Policy, The National Council on the Aging Inc.

RILEY, M.W. (1994). "Aging and Society: Past, Present, and Future », The Gerontologist, vol. 34, n' 4, p. 436-446.

RILEY, M.W. (1976). "Age Strata in Social Systems", dans R.H. Binstock et E. Shanas (dir.), Handbook of Aging and the Social Sciences, New York, Van Nostrand Reinhold, p. 189217. Voir aussi la version 1985 de ce texte (même éditeur).

RILEY, M.W., M. JOHNSON et A. FONER (1972). Aging and Society, vol. 3, "A Sociology of Age Stratification », New York, Russel Sage Foundation.

ROCHER, G. (1969). Introduction à la sociologie générale, tomes I, II et III, Montréal, Hurtubise/HMH.

ROSE, A.M. (1965). "The Subculture of the Aging: A Framework for Research in Social Gerontology ", dans A.M. Rose et W.A. Peterson (dir.), Old People and Their Social World, Philadelphia, F.A. Davis \& Co., p. 3-16.

Rosow, I. (1967). Social Integration of the Aged, New York, The Free Press.

Rosow, I. (1974). Socialization to Old Age, Los Angeles, University of California Press.

RoY, J., A. Vézina et A. Cliche (1996). Les aînés et les solidarités dans leur milieu: l'envers du mythe, Étude portant sur la solidarité horizontale des personnes âgées vivant à domicile et résidant dans le territoire du CLSC La Source, Québec, Centre de recherche sur les services communautaires de l'Université Laval.

SCHARLACH, A.E. (1988). "Peer Counselor Training for Nursing Home Residents », The Gerontologist, vol. $28, n^{\circ} 4$, p. 499-502. 
SCOTT, W.J. et H.G. GRASMICK (1979). "Generations and Group Consciousness, A Quantification of Mannheim's Analogy », Youth and Society, vol. 11, $\mathrm{n}^{\circ} 2, \mathrm{p} .191-213$.

SIMARD, C., M.-A. DELISLE, D. DROUIN et R. LAROUCHE (1992). Rapport d'étape, Projet de recherche CREDO du CLSC ArthurCaux, Sainte-Foy et Laurier Station, Département d'éducation physique de l'Université Laval et CLSC Arthur-Caux, tomes 1, 2 et 3.

STRYCKMAN, J. (1982). Mariages et mises en ménage au cours de la vieillesse, Québec, Laboratoire de gérontologie sociale de l'Université Laval.

TOUSSAINT, F. (1985). Les conditions de vie des retraités de l'enseignement du Québec, Rapport d'une enquête conduite en juin 1985, Québec, Association des retraités de l'enseignement du Québec.

TOUSSAINT, F. (1988-1989). L'entraide dans les rangs de l'AREQ, Rapport sur la situation en 1988-89, Québec, Assoc. des retraités de l'enseignement du Québec. Coll. Instruments, $n^{\circ} 3$.

WARREN, R.L. (1963). Community in America, Chicago, Rand McNally \& Co.

WEST, G.E., M.-A. DELISLE, C. SIMARD et D. DROUIN (1996). "Leisure Activities and Service Knowledge and Use among the Rural Elderly », Journal of Aging and Health, vol. 8, $n^{\circ} 2$, p. 254-279.

WILLEMS, E. (adapté par A. Cuvillier) (1970). Dictionnaire de sociologie, Paris, M. Rivière et Cie.

WILLIAMS, A. (1983). "Changing Patterns of Horizontal and Vertical Linkages ", dans R.T. Coward et W.T. Smith, Family Services. Issues and Opportunities in Contemporary Rural America, Lincoln and London, University of Nebraska Press, p. 87-101.

ZAY, N. (1984). "Impact de la gérontologie sur la culture », dans Questions de culture $n^{\circ} 6$, Québec, Institut québécois de recherche sur la culture, p. 81-99.

ZAY, N. (1981). Dictionnaire-manuel de gérontologie sociale, Québec, Presses de l'Université Laval, 767 p. 